experience of and possesses a profound knowledge about hysteria as an expression of organic brain syndromes and yet he has the ability to portray such symptomatology as serving socially adaptive functions. Thus, it is refreshing to encounter his respect for the psychoanalytic search for experiential mechanisms as a basis for hysterical personality structure and symptomatology.

Subjects such as deliberate disability, including self mutilation and compensation neurosis, are examined in relation to classical conversion hysteria, hypochondriasis, pain and epidemic hysteria. The propensity for developing such symptomatology on the basis of personality structure is examined in terms of the range of normal central nervous system constitution. There is a comprehensive and well integrated bibliography. This is a book which will really help the clinician and which commands the attention of all those researching the field.

\section{Bedside Diagnosis}

By Charles Seward and David Mattingly. 11 th edn. Pp. $x i+357$, illustrated. Churchill Livingstone, Edinburgh, London and New York, 1979. £7.95.

The 11th edition of this small book sets out to tackle the problem of diagnosis by emphasizing that any one symptom may arise from a multiplicity of organs.

Therefore, in each chapter, the authors take one major symptom or sign and give a synopsis of diseases which may give rise to it. They go on to discuss the basic physiology underlying such a symptom and then give a description of each disease mentioned in the synopsis stating, in each case, the associated clinical signs and investigations which may prove useful in establishing the diagnosis.

However, since one disease, e.g. cholelithiasis, can give rise to several symptoms, e.g. chest pain, epigastric pain, vomiting, pyrexia and jaundice, it will be briefly discussed in several sections. The authors have tried to avoid repetition by describing any one disease in just one section but extensive cross-referencing then arises as the reader is directed from chapter to chapter.

Its small size and comprehensive lists will no doubt be of tremendous value to the new clinical medical student who, bewildered by the symptoms he is confronted with on the wards, will be able to reach into his pocket for quick and easy reference.

\section{Clinical Hypertension}

By J. D. Swales. Pp. xii +207 , illustrated. Chapman and Hall, Andover, 1979. £6.50 (hardback £10.50).

Professor Swales, in his book on hypertension attempts to cover the broad scope of hypertension relevant for the clinical doctor; discussing areas of controversy with a balanced judgement and thus provides an easily readable book for the practising specialist in many disciplines.

The physiological background to hypertension and the pathology resulting from hypertension is described. The clinical features of hypertension pertinent to surgical and medical treatment including obstetric aspects of hypertension are discussed. References are kept to a minimum at the end of each chapter with a list of recent reviews for further reading. As with any subject presented by a clinician, personal points of view sometimes prevail, but overall, Professor Swales has given a balanced clinical review of this subject.

This is an easily readable book and can be highly recommended for the postgraduate doctor during his early qualification years and also for the general practitioner requiring an update in this subject. This book comes at an appropriate time when there have been many advances, both in the medical treatment and investigation of the hypertensive patient. It is reasonably priced and can be well recommended 3

\section{A Colour Atlas of Endocrinology}

By R. Hall D. Evered and R. Greene. Pp. 176, illus trated. Wolfe Medical Publications, London, 1979. £18.00

Perhaps one of the most fundamental attractions of endocrin ology lies in the fact that many disorders can be recognized at a glance. The authors of this atlas have fully exploited this aspect of the subject and have compiled a splendid collection of clinical photographs illustrating a wide range of conditions. The longest, and probably the most successful, section is on thyroid disorders but pituitary and adrenal conditions are alsowell covered. The chapters on disorders of growth and sexual development are less successful, as are the sections $\vec{\Delta}$ on diabetes, parathyroid disease, and gut hormones. The general standard of the colour photographs is good, an most have been well chosen although there is a certain amount of overlap between different chapters.

The authors have provided brief accounts of the saliente clinical features shown in the photographs, together with tables and figures illustrating the underlying hormonal an\& metabolic disturbances. While these contain a number of 'pearls', they are too brief to be particularly helpful and thew authors advise that the atlas should be used in conjunctiono with a conventional textbook of endocrinology. When used' in this role the atlas could well add spice to some of theo weightier textbooks for medical students or nurses. Even? case-hardened physicians may enjoy browsing through it $\vec{Z}$ It can be read quite easily in an evening and could be in valuable in the last minute brush-up for finals or member ship.

\section{A Colour Atlas of Pathogenic Fungi}

By D. Frey, R. J. Oldfield and R. C. Bridger. Pp. 168 illustrated. Wolfe Medical Publications, London, 1979 f16.00.

The identification of fungi in routine diagnostic laboratorieso frequently causes many difficulties. Although successfu且 cultures may be made, the colonial morphology and micro scopic appearances are a frequent source of confusion.

Colonial morphology is well shown, although it would have been helpful for many isolates if the reverse side of the? culture was also illustrated. The microscopic appearances of the cultures are well shown in most of the photomicrographs.

There is, however, a transposition error in that Figure 37.2을 shows Favic chandeliers and 35.2 Reflexive branching. The histology sections clearly show the characteristic lesions.

The tables at the end provide a useful summary of cultural characteristics. A comprehensive list of references is provided for those workers who wish to obtain further information.

Decision Making in Medicine-the Practice of its Ethics
Edited by GoRDON ScORER and ANTHONY WING. Pp. 211 을.
Edward Arnold, London, 1979. £6.50. This book offers guidance for those who have to make $N$ difficult ethical decisions in their daily practice. Chapters deal with genetic counselling and genetic engineering; contraception and sterilization; termination of pregnancy; sex education; euthanasia; child deformity and handicap; $\sigma$ decisions about dying and death; use and misuse of drugs; and safeguards in clinical research. The moral issues are those that all in clinical practice face daily. There is no other $\bar{\varnothing}$ book that faces these issues so squarely and provides helpful? guidelines not only for doctors but for the large and growing army of paramedicals who help to share the burden by making wise decisions in difficult circumstances. 